\title{
Irreproducible results in preclinical cardiovascular research: opportunities in times of need
}

\author{
Karin R. Sipido* \\ Department of Cardiovascular Sciences, KU Leuven, Campus Gasthuisberg, Herestraat 49, B-3000 Leuven, Belgium
}

\section{A hot topic}

Concerns about the lack of reproducibility in science have become a mainstream topic ${ }^{1}$ and were centre-stage of a well-attended session at the ESC congress in Munich. ${ }^{2}$ In medical research, lack of reproducibility is a bottleneck in translation. In 2011, Bayer Healthcare examined 67 biomedical research studies and could reproduce the main results in only 5 of them. ${ }^{3}$ The problem is not limited to cardiovascular research, but given disappointment in translation of some very promising concepts, it is an urgent question.

So what is behind this failure of reproducibility? Fraud, manipulation, sloppy science - or is there a fundamental issue in the nature of research to consider.

\section{Fraudulent or false data: the magnitude of the problem and ways to look at it}

Fraud and manipulation in reporting research results have taken centre stage in many debates on irreproducibility and scientific integrity. Several studies have attempted to quantify the extent and prevalence of scientific misconduct, based on surveys and metadata on retracted articles.

Surveys in the scientific community have suggested that lack of honesty, varying from minor to major misconduct, is rather widespread. ${ }^{4}$ The meta-analysis concluded: 'However, it is likely that, if on average $2 \%$ of scientists admit to have falsified research at least once and up to $34 \%$ admit other questionable research practices, the actual frequencies of misconduct could be higher than this.'

Serious flaws in published data lead to retraction and in 2012 Grieneisen and Zhang ${ }^{5}$ published an analysis of retractions across all scientific disciplines. Retractions are proportional to the share of the discipline in the literature. Medicine scores high, representing $50 \%$ of all retractions, and, with medicine accounting for $40 \%$ of published papers, this is slightly above the mean expected rate. The underlying reasons for retractions are predominantly questionable data with some $20 \%$ of all retractions cases the result of serious research misconduct, i.e. fraudulent of fabricated data, and another $26 \%$ of questionable data. Plagiarism is another major reason.
As a fraction of all publications, retractions still remain limited (at $0.02 \%$ noted in 2012), though this may reflect a level of failure to detect and pursue errors. Of note, behind these numbers of retracted papers, there are a smaller number of offenders. Fifteen repeat offenders account for $52 \%$ of all retractions in the period between 1980 and 2010. Medicine is prominently present in this list, with one author associated with a set of 88 papers retracted because of lack of ethics approval by the institutional review board. A very recent analysis of retractions based on the data platform Retraction Watch and reported in Science, underscores that the number of retractions has levelled off, at about 4 per 10000 published papers. ${ }^{6}$ Another commentary however justly points out the danger of serious misbehaviour in medicine. ${ }^{7}$

Recognition of the impact of research misconduct has motivated institutions, funders, and governments to set up investigative panels, linked to severe penalties for offenders. ${ }^{8}$

\section{An editor's perspective on misconduct}

Journals and the editorial review process are gatekeepers to prevent publication of flawed data. In the 5 years that our team was leading Cardiovascular Research, ${ }^{9}$ we have kept a close watch and have kept logs for quantification. Serious misconduct with fraudulent data, image manipulation or wrongful reporting, was rare. Out of 6500 submissions, 31 were rejected because of plagiarism and 60 papers with suspicion of incorrect data reporting or manipulation were investigated. Out of these, 15 were shown to be errors. All together, we detected serious misconduct in $0.1 \%$ of all submissions. The ESC Journals Ethics Committee has been most helpful in addressing some of these cases. ${ }^{10}$

Much more common was what is referred to as 'sloppy science'. Flaws in study design, low sample numbers, inappropriate statistics, incomplete description of methods, lack of transparency in results reporting and overstretched conclusions undermine the solidity and robustness of data, and hence their reproducibility. Similar problems have been noted by others, e.g. ${ }^{11}$ We therefore set out editorial policies $^{12}$ translated in instructions to authors and editorial comments, ${ }^{13}$ to guide authors in what was expected from a study. The framework thus created continues to guide the present editorial team. ${ }^{14}$

* Corresponding author. Tel: 3216 330815; fax: 3216 345844, E-mail: Karin.Sipido@kuleuven.be

Published on behalf of the European Society of Cardiology. All rights reserved. @ The Author(s) 2019. For permissions, please email: journals.permissions@oup.com. 


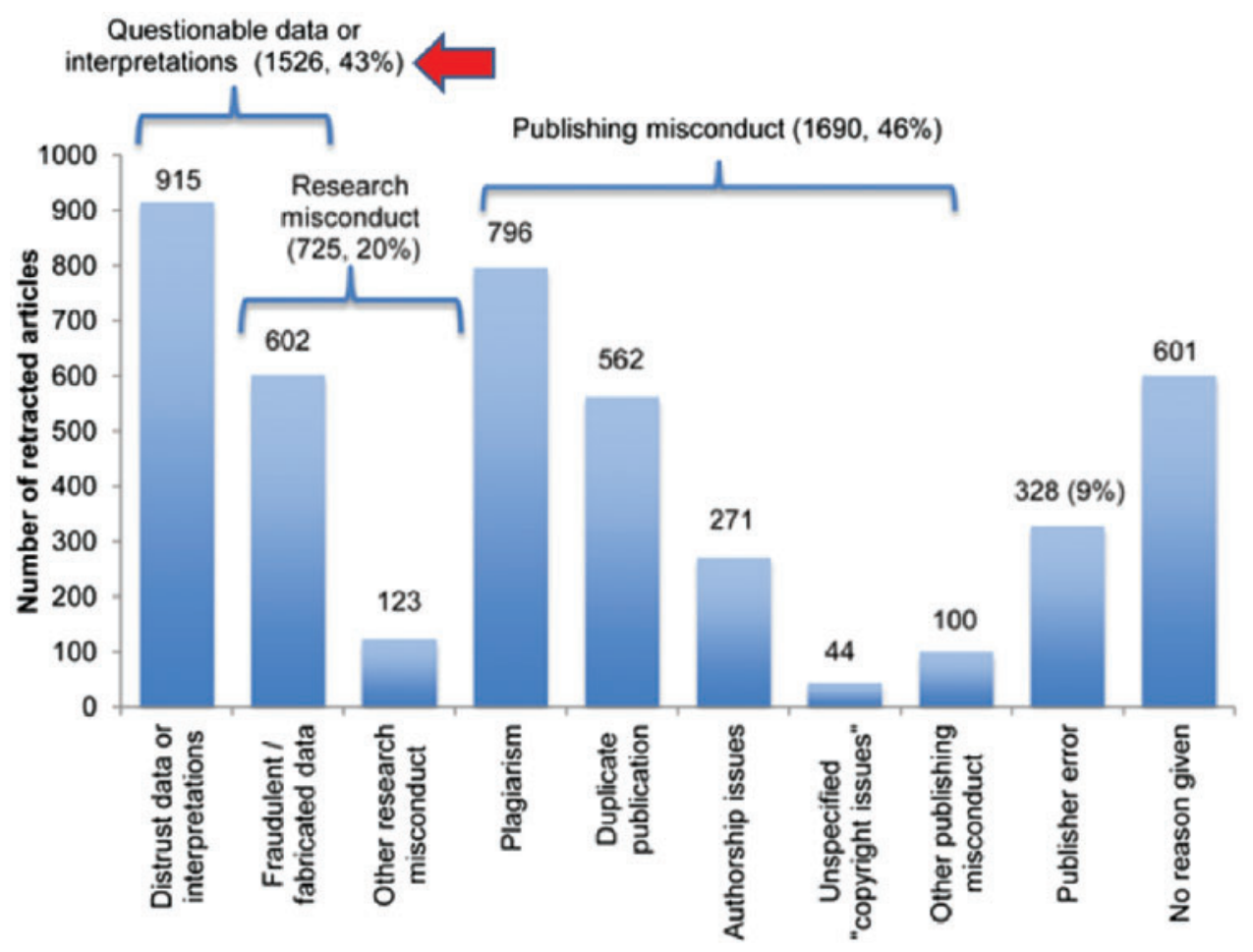

Figure I Analysis of reasons accounting for retraction. Data from Grieneisen and Zhang. ${ }^{5}$

\section{The uncertainty of life and science}

Whereas speculation is a natural part of scientific discussion, the perspective to potential new cures has become a too common ingredient of the conclusions of many a preclinical study.

Life is an oddity in nature and can only be sustained at great cost against the laws of thermodynamics. 'One can define Life to be an ordered system that can sustain itself against the tendency to disorder' was stated by Stephen Hawking (www.hawking.org.uk/life-in-the-universe. html). Statistics remain a calculation of chance and the odds of having performed a fool-proof experiment in biology are small. Uncertainty remains and many experiments in biology are hypothesis-generating. Fraudulent and poor scientific practice can be remedied, but accepting reality and relativity of findings is another important element in addressing the current 'perceived crisis'.

\section{Opportunities for better science}

The call for action to improve reproducibility of results creates opportunities for better quality science and for adopting novel approaches in preclinical research.

The session at the ESC congress was a stimulating event with thoughtful presentations on how to improve the quality of preclinical research. These included the lessons learnt from cell and gene therapy, and a debate on models for disease. The scientific community can set out guidelines for research methodology through position papers, as e.g. done by the community studying cardioprotection in Cardiovascular Research, ${ }^{16}$ embrace more rigorous quality control and provide critical reviews in fast moving fields. ${ }^{17}$ Guidance for design, statistics, and reporting in experimental research can be readily found - the challenge is to apply the proper approaches as they come at a cost. ${ }^{11}$

Working together has many benefits. Competition in science is of all times ${ }^{18}$ and can be a healthy stimulus but collaborative research is very impactful, not only in clinical but also in basic and preclinical research. ${ }^{19}$ Shared practices and shared data can enhance quality, and can optimize use of existing data. Closer interactions between clinical and basic science could lead to adopting of some sound practices from clinical research. These include randomization, blinding and multi-centre research. The registration of preclinical research and the platform created (preclinicaltrials.eu) is an example. In neurology and stroke research, longer-standing initiatives for optimization of preclinical research now include the CAMARADES platform (http://www.dcn.ed.ac.uk/camarades/) for larger-scale approaches.

The call for 'Open Science' is strong, to some extent with an economic perspective to stimulate innovation, but also as a mechanism to enhance quality of science. Technology for large data platforms will come into reach and is one of the priorities in the EU research agenda (https://ec.eu ropa.eu/research/openscience/index.cfm?pg=open-science-cloud). For life sciences, several platforms exist, as recently reviewed ${ }^{20}$ and structuring data platforms in Europe to facilitating data sharing and access is the major task of ELIXIR (https://www.elixir-europe.org/). Indiscriminate 'dumping' of raw data is unlikely to work and a serious exercise should look into the feasibility and cost:benefit of raw data sharing. Tackling issues of privacy and ensuring data quality are but some of the hurdles to be taken in particular in clinical research, but there is also great potential in sharing clinical experience. ${ }^{21}$ Therefore, overcoming these obstacles, 
optimizing and refining access to data, can multiply novel insights from experimental and clinical studies. Availability of data will still require analysis and placing findings in context, present and future, but publication practice will adapt to more open data platforms.

Learned societies and their journals, academic and research institutions, funders and the public are stakeholders in better science. Inspirational quotes on the ESC congress bags included an appropriate saying by Amelia Earhart: 'The most difficult thing is the decision to act, the rest is mere tenacity'. The present editorial team will pursue and expand the journal's commitment to highest quality. ${ }^{14}$

Conflict of interest: none declared.

\section{References}

1. Fanelli D. Opinion: is science really facing a reproducibility crisis, and do we need it to? Proc Natl Acad Sci USA 2018;115:2628-2631.

2. Schmidt C, Voigt N. ESC Congress 2018 highlights in basic science: a report from the Scientists of Tomorrow. Cardiovasc Res 2018;114:e103-e1e5.

3. Prinz F, Schlange T, Asadullah K. Believe it or not: how much can we rely on published data on potential drug targets? Nat Rev Drug Discov 2011;10:712.

4. Fanelli D. How many scientists fabricate and falsify research? A systematic review and meta-analysis of survey data. PLoS One 2009;4:e5738.

5. Grieneisen ML, Zhang M. A comprehensive survey of retracted articles from the scholarly literature. PLoS ONE 2012;7:e44118.

6. Brainard J. Rethinking retractions. Science 2018;362:390-393.

7. Marcus A. A scientist's fraudulent studies put patients at risk. Science 2018;362:394.

8. Kornfeld DS, Titus SL. Stop ignoring misconduct. Nature 2016;537:29-30.

9. Sipido KR. The generosity of the Cardiovascular Research community. Cardiovasc Res 2017;113:1701-1702.
10. On behalf of all Committee Members, Simoons ML. The ESC Publications Ethics Committee: report from the first 5 years' experience of the European Society of Cardiology (ESC) journals family ethics committee. Eur Heart J 2018;39:2518-2521.

11. Eisner DA. Reproducibility of science: fraud, impact factors and carelessness. J Mol Cell Cardiol 2018;114:364-368.

12. Sipido KR, Casadei B, Holvoet P, Janssens S, Luttun A, Sampaolesi M. Bedside to bench: a look at experimental research with a clinical trial checklist. Cardiovasc Res 2014;101:1-3.

13. Sipido KR, Gal D, Luttun A, Janssens S, Sampaolesi M, Holvoet P. Peer review: (r)evolution needed. Cardiovasc Res 2017;113:e54-ee6.

14. Guzik TJ, Antoniades C, Baker AH, Harrison DG, Loughrey CM, Maffia P, Murphy E, Nicklin SA, Peter K, Pearson J, Casadei B. What matters in Cardiovascular Research? Scientific discovery driving clinical delivery. Cardiovasc Res 2018;114:1565-1568.

15. De Boeck P, Jeon M. Perceived crisis and reforms: issues, explanations, and remedies. Psychol Bull 2018;144:757-777.

16. Lecour S, Botker HE, Condorelli G, Davidson SM, Garcia-Dorado D, Engel FB, Ferdinandy P, Heusch G, Madonna R, Ovize M, Ruiz-Meana M, Schulz R, Sluijter JPG, Van Laake LW, Yellon DM, Hausenloy DJ. ESC working group cellular biology of the heart: position paper: improving the preclinical assessment of novel cardioprotective therapies. Cardiovasc Res 2014;104:399-411.

17. Perrino C, Barabási A-L, Condorelli G, Davidson SM, De Windt L, Dimmeler S, Engel FB, Hausenloy DJ, Hill JA, Van Laake LW, Lecour S, Leor J, Madonna R, Mayr M, Prunier F, Sluijter JPG, Schulz R, Thum T, Ytrehus K, Ferdinandy P. Epigenomic and transcriptomic approaches in the post-genomic era: path to novel targets for diagnosis and therapy of the ischaemic heart? Position Paper of the European Society of Cardiology Working Group on Cellular Biology of the Heart. Cardiovasc Res 2017; 113:725-736.

18. Watson JD. The Double Helix: A Personal Account of the Discovery of the Structure of DNA. USA/UK: Atheneum/Weidenfeld \& Nicolson; 1968.

19. Gal D, Glänzel W, Sipido KR. Mapping cross-border collaboration and communication in cardiovascular research from 1992 to 2012. Eur Heart J 2017;38:1249-1258.

20. Rigden DJ, Fernández XM. The 2018 Nucleic Acids Research database issue and the online molecular biology database collection. Nucleic Acids Res 2018;46:D1-D7.

21. Kiley R, Peatfield T, Hansen J, Reddington F. Data sharing from clinical trials-a research funder's perspective. N Engl J Med 2017;377:1990-1992.

\section{Author}

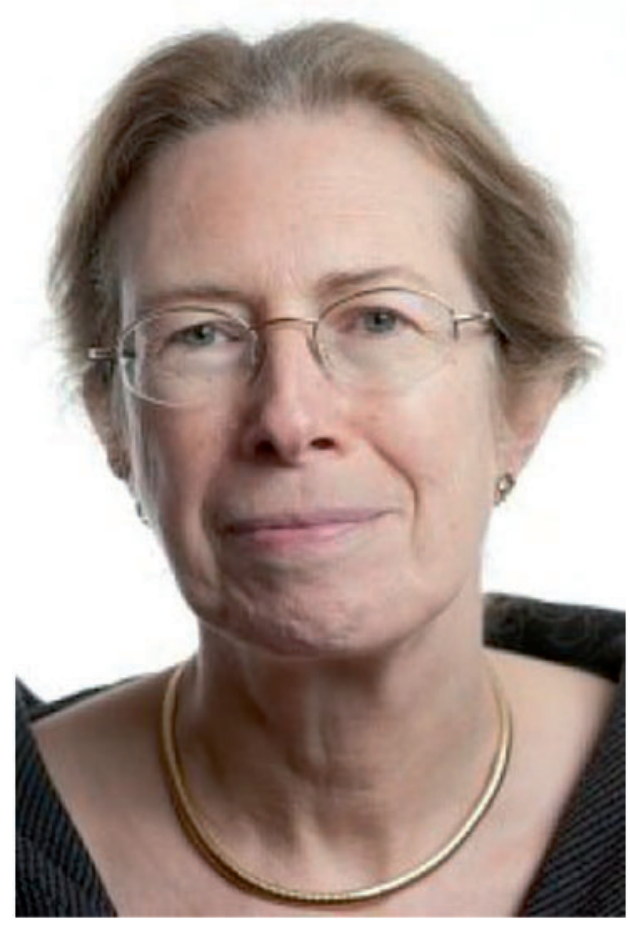

Biography: Karin Sipido is Professor of Medicine and Head of Experimental Cardiology at the KU Leuven, the University of Leuven, Belgium. She holds an MD degree, trained in Internal Medicine and Cardiology and holds a PhD in Physiology. Her academic research focuses on cellular mechanisms of heart failure and arrhythmias. With collaborators in cardiac imaging and clinical electrophysiology, the research receives a broader clinical perspective. She is editorial board member of several leading cardiovascular journals; she was Associate Editor of the European Heart Journal and Editor-in-Chief of Cardiovascular Research 2013-2017. She was elected member of the Academia Europaea, Fellow of the European Society of Cardiology, Fellow of the American Heart Association and of the International Society for Heart Research. She assumed several tasks within the European Society of Cardiology. She was founding member and President of the Alliance for Biomedical Research Europe 2013-2015. Currently she chairs the Scientific Panel for Health under the provision of the European Commission H2020 program. Within the KU Leuven, she chairs the Council for Research Strategy. 